\title{
Un dispositif d'accompagnement dans la transition lycée-université (IUT) : enjeux et effets
}

Isabelle Bournaud and Patrick Pamphile

\section{(2) OpenEdition \\ 12 Journals}

\section{Electronic version}

URL: http://journals.openedition.org/ripes/3156

DOI: 10.4000/ripes.3156

ISSN: 2076-8427

\section{Publisher}

Association internationale de pédagogie universitaire

\section{Electronic reference}

Isabelle Bournaud and Patrick Pamphile, "Un dispositif d'accompagnement dans la transition lycéeuniversité (IUT) : enjeux et effets", Revue internationale de pédagogie de l'enseignement supérieur [Online], 37(2) | 2021, Online since 15 March 2021, connection on 18 March 2021. URL: http:// journals.openedition.org/ripes/3156 ; DOl: https://doi.org/10.4000/ripes.3156

This text was automatically generated on 18 March 2021.

Article L.111-1 du Code de la propriété intellectuelle. 


\title{
Un dispositif d'accompagnement dans la transition lycée- université (IUT) : enjeux et effets
}

\author{
Isabelle Bournaud and Patrick Pamphile
}

\section{Introduction}

1 Les difficultés sous-jacentes à la transition entre le lycée et l'université ont fait l'objet de nombreux travaux (cf. Paivandi, 2015 pour une synthèse). Ces travaux montrent que les difficultés disciplinaires ne sont pas les seules causes d'échec en première année à l'université (Berthaud, 2017; Michaut et Romainville, 2012). Ainsi, si la démocratisation de l'accès à l'enseignement supérieur a eu pour conséquence une hétérogénéité des étudiants en matière de prérequis, on constate également une très grande hétérogénéité de leurs méthodes de travail et de projets professionnels (Michaut et Romainville, 2012; Paivandi, 2015). En particulier, bon nombre d'étudiants à l'entrée à l'université ne maîtrisent pas la capacité à adopter une posture réflexive de gestion de ses processus mentaux, et à analyser et à réguler sa propre cognition, facteur reconnu important pour la réussite (cf. Dupont et al., 2015; Paivandi, 2015, pour des revues de littérature).

2 Le plan Réussite en Licence, lancé fin 2007 en France, prévoyait ainsi de renforcer les compétences des étudiants, notamment en termes de méthodes de travail et d'autonomie ${ }^{1}$. Différents dispositifs à destination des primo-entrants ont été mis en œuvre dans ce cadre entre 2007 et 2012, variant selon les universités et au sein même des universités ${ }^{2}$. Ces dispositifs diffèrent en matière de contenu (préparation à la vie professionnelle, orientation active et encadrement des étudiants), de modalités d'organisation (ateliers, cours magistraux, ...) et de bénéficiaires (s'adressent à tous les étudiants ou réservés à ceux en difficultés) (cf. Perret 2014; Raucent et al., 2010). Si les actions mises en place ne semblent pas avoir eu d'effets négatifs, l'analyse de leur efficacité est difficile au regard des objectifs affichés par les équipes pédagogiques: 
développement de l'autonomie, de la métacognition, élaboration d'un projet professionnel... Néanmoins, le constat partagé est que l'adhésion aux dispositifs ne fait pas toujours l'unanimité tant chez les étudiants, dont les plus en difficulté ne se sentent pas concernés, que chez les enseignants qui les perçoivent comme une charge supplémentaire dans leur enseignement.

3 La problématique qui motive nos travaux est celle de la réussite en première année universitaire. Un des moyens de faciliter la transition lycée-université consiste à sensibiliser tous les primo-entrants à l'université sur le changement de paradigme éducatif - dans lequel l'étudiant se voit confier un rôle plus actif dans la construction de ses connaissances et le développement de ses compétences (Lison et Jutras, 2014) et à les accompagner dans l'engagement dans leur formation et le développement de l'autonomie dans leurs apprentissages. C'est dans ce contexte que se situe l'approche que nous proposons.

4 La première partie de cet article présente le cadre théorique de ce travail. La deuxième partie décrit le dispositif retenu et sa mise en œuvre dans le cadre d'une formation de 179 étudiants en $1^{\text {re }}$ année universitaire. La troisième partie présente une évaluation de ce dispositif, selon plusieurs indicateurs: les résultats académiques au semestre 1 (validation/échec, abandon) et la perception de maîtrise des étudiants de leurs méthodes de travail.

\section{Cadre théorique}

5 L'accompagnement a fait l'objet de nombreux travaux dans l'enseignement secondaire (cf. Reverdy, 2017 pour une perspective historique). En revanche, les travaux dans le supérieur sont assez récents (Perret, 2015; Raucent et al., 2010). Les dispositifs d'accompagnement mis en œuvre en licence sont destinés à réduire l'échec des étudiants en facilitant la transition entre le lycée et l'enseignement supérieur (Cosnefroy et al., 2014). Ils ont quatre principaux objets : la construction d'un projet professionnel, l'appropriation de contenus (soutien disciplinaire), la méthodologie du travail universitaire, ou l'affiliation au monde universitaire (accueil des primoentrants, enseignants référents, contrôle continu, ...).

6 Selon Verzat (2010), «[1]'accompagnement se présente comme un processus clé permettant d'entrainer les étudiants dans une démarche réflexive pour apprendre à partir de l'expérience et développer des savoir-être autonomes" (p.44). On peut distinguer deux registres dans les pratiques d'accompagnement mobilisées : guider registre du conseil et de l'orientation, permettre à la personne de trouver sa voie - et conduire - registre de la formation, exercer une autorité (Paul, 2009a). En pratique, ces différents registres sont articulés au regard de la situation considérée, et donnent lieu à des rôles d'accompagnant différents. Pour Cosnefroy et al. (2014), lorsque le registre conduire est trop privilégié au détriment du registre guider cela nuit à un accompagnement de type méthodologique.

7 On peut également caractériser une action d'accompagnement par la fonction principale poursuivie: préventive (installer chez l'étudiant des habilités estimées nécessaires à la réussite universitaire), remédiative (face à des lacunes constatées) ou formative (accompagner dans l'acquisition de compétences professionnelles) (cf. Noël et Romainville, 1998). 
Une première question qui a orienté ce travail est la suivante : Comment accompagner la transition lycée-université de primo-entrants: quel contenu? Qui accompagne? Quelle fonction et quelle forme d'accompagnement?

Dans ce travail, nous présentons un accompagnement d'étudiants à leur entrée dans une formation universitaire, en $1^{\text {re }}$ année de DUT (Diplôme Universitaire de Technologie). L'approche retenue est préventive, elle vise à faciliter la transition lycéeuniversité en amenant les étudiants à avoir une posture réflexive sur leurs méthodes de travail, et en les outillant afin qu'ils soient plus efficaces dans leurs apprentissages disciplinaires. Elle repose sur des ateliers, et sur diverses activités pédagogiques déployées dans les enseignements disciplinaires.

Lors de la mise en place d'un dispositif pédagogique, il est indispensable d'évaluer celui-ci afin, d'une part de l'améliorer et de le valoriser (Bernard, 2011), et également de rendre compte aux différentes parties prenantes (étudiants, enseignants, institution l'ayant financé...). Les travaux sur l'évaluation des actions d'accompagnement pédagogiques sont nombreux (Salmon et al., 2009). Ils mettent en évidence qu'une telle évaluation est complexe et difficile à analyser (Michaut, 2003; Perret, 2015). Les difficultés résident notamment dans le choix des critères d'évaluation de l'action (validation semestre, développement de méthodes de travail, affiliation de l'étudiant, ...), dans le recueil de données représentatives, dans le choix de l'outil de recueil (questionnaire à questions fermées, ouvertes, entretiens, ...) ou encore le moment où l'évaluation est réalisée (à chaud ou à froid) (cf. Salmon et al., 2009). De surcroit, une difficulté inhérente aux recherches en éducation est que la validité de l'évaluation peut être remise en question : de très nombreuses variables interviennent dans la réussite étudiante, elles peuvent varier d'une année à l'autre, ou ne sont pas toutes considérées simultanément (Sanchez et Monod-Ansaldi, 2015).

11 Une deuxième question que nous avons abordée est ainsi la suivante : Comment évaluer le dispositif d'accompagnement proposé? Nous avons choisi d'évaluer le dispositif mis en place au regard, d'une part des résultats académiques au semestre 1, et d'autre part de l'évolution de la perception de maîtrise des étudiants (Bournaud et Pamphile, 2020).

\section{Quel contenu d'accompagnement, qui accompagne et comment?}

12 Le but du dispositif que nous proposons est de lier les stratégies d'enseignement aux stratégies d'apprentissage afin de mobiliser les étudiants sur le long terme, et d'engager les enseignants dans la démarche, sans alourdir la charge de travail de ces derniers. Il s'agit, comme le préconise Tinto (2006, cité dans Philion et al., 2010, p. 4), que l'accompagnement fasse partie intégrante de la formation.

13 Pour les primo-entrants d'une formation, "étudiants novices", il est important de connaitre les difficultés rencontrées par leurs prédécesseurs au cours de leur première année universitaire : cela leur permet de se projeter plus aisément dans la formation. Par ailleurs, si les enseignants ont connaissance, en amont, des difficultés non disciplinaires mais qui peuvent pénaliser l'étudiant dans ses apprentissages, ils seront plus en mesure de l'aider dans ses apprentissages. Par conséquent, nous avons mené en 2018, une étude afin de mettre en évidence le lien entre les méthodes de travail des étudiants du DUT Gestion des Entreprises et des Administrations (GEA ${ }^{3}$ et leurs 
résultats académiques au premier semestre (S1) (Bournaud et al., 2018). Cette étude montre qu'un étudiant en échec au S1 est en échec dans toutes les matières, alors qu'un étudiant en pleine réussite (moyenne au S1 supérieure à 12/20) est en réussite dans toutes les matières. Cet « effet de taille » dans l'analyse statistique des résultats montre qu'il n'y a pas qu'un effet disciplinaire et donc qu'un soutien disciplinaire pour les étudiants en difficultés n'est pas suffisant. Nous avons ensuite caractérisé les étudiants en réussite et les étudiants en échec à partir d'une enquête sur leurs méthodes de travail déclarées. Les étudiants en échec se distinguent par des méthodes de travail reconnues comme peu adaptées à l'université (Paivandi, 2015), à l'inverse des étudiants en pleine réussite (voir Tableau 1).

Tableau 1. Caractérisation des étudiants en échec/en réussite au DUT GEA

\begin{tabular}{|c|c|}
\hline Etudiants en échec « Novice» & Etudiants en réussite « Expert » \\
\hline $\begin{array}{l}\text { Déclarent moins souvent (que la } \\
\text { population): }\end{array}$ & Déclarent plus souvent : \\
\hline $\begin{array}{l}\text { - planifier leur travail et travailler } \\
\text { régulièrement } \\
\text { - qu'être actif en TD est important pour les } \\
\text { apprentissages } \\
\text { - être motivé par la formation }\end{array}$ & $\begin{array}{l}\text { - que les évaluations formatrices sont importantes } \\
\text { pour les apprentissages } \\
\text { - faire des exercices d'entrainement } \\
\text { - préparer les TD à l'avance } \\
\text { - avoir peu de difficultés à se concentrer plusieurs } \\
\text { heures }\end{array}$ \\
\hline Ont un temps de transport plus élevé & \\
\hline
\end{tabular}

14 L'objectif de notre étude était avant tout de permettre aux primo-entrants, mais aussi aux enseignants, de prendre conscience, en amont, de l'importance des méthodes de travail dans la réussite.

15 A partir de cette étude, et en s'appuyant sur le travail de Cosnefroy et al. (2014), nous avons, en équipe pédagogique, élaboré un dispositif d'accompagnement des primoentrants. Cette démarche d'élaboration en équipe d'un dispositif pédagogique s'inscrit dans la lignée du modèle de la dynamique innovationnelle puisqu'il s'agit d'impliquer l'enseignant dans un processus collectif d'amélioration autour d'une problématique commune (Lison et al., 2014).

16 Le dispositif proposé repose d'une part sur des ateliers obligatoires pour tous les étudiants en début d'année, et d'autre part sur diverses activités pédagogiques déployées dans les enseignements disciplinaires. En effet, les étudiants ont besoin de percevoir l'utilité du dispositif afin d'y adhérer, et également de temps pour s'approprier des méthodes de travail. Il était donc nécessaire de construire un continuum entre les ateliers et les enseignements disciplinaires afin de maintenir l'engagement des étudiants, et d'associer au dispositif les enseignants pour faire écho au contenu des ateliers dans les enseignements disciplinaires. Les ateliers et les activités pédagogiques choisies sont présentés ci-après. 


\subsection{Les ateliers}

17 Les ateliers visent à rendre l'étudiant autonome dans ses apprentissages en lui proposant de réfléchir, en petits groupes, à des méthodes de travail appropriées aux exigences d'une formation universitaire. Ils sont obligatoires. Ce choix a été guidé par les travaux qui montrent que les dispositifs d'accompagnement, lorsqu'ils sont facultatifs, touchent rarement les étudiants qui en auraient le plus besoin (Perret et Morlaix, 2015).

\subsubsection{Objectifs}

18 Un des premiers objectifs des ateliers est de permettre aux étudiants de s'interroger sur les facteurs de réussite/échec lors de la transition lycée-université. Plus précisément, l'étudiant va être amené à réfléchir sur les différences entre le lycée et l'université, et sur les méthodes de travail qu'il utilisait au lycée. Il s'agit de faire en sorte que l'étudiant prenne conscience qu'il a les moyens d'agir sur sa réussite, qu'il n'y a pas une seule bonne méthode mais différentes méthodes, et que pour réussir il faut savoir choisir la plus appropriée au contexte (Villeneuve et al., 2010).

19 Les thèmes des ateliers ont été choisis au regard des résultats de l'étude menée en 2018 et des travaux sur la réussite étudiante. Dupont et al. (2015) rapportent notamment une méta analyse qui montre que le soutien à la réussite apporté par l'institution est efficace lorsqu'il se concentre sur l'acquisition de compétences et connaissances considérées comme favorisant la réussite des étudiants (mémorisation, gestion du temps, prise de notes). Les thèmes choisis sont les suivants :

20 - Connaître son environnement et les ressources disponibles; rédiger un mail professionnel.

21 - Gérer son temps, s'organiser, planifier (Houart, 2017).

22 - Connaître le fonctionnement de la mémoire (mémoire de travail/ mémoire à long terme, inhibition, ...), la plasticité du cerveau (Houdé, 2011) et les types d'apprentissage - surface/profondeur (Biggs, 2011).

23 - Distinguer les trois type de connaissances (déclarative/ procédurale/ conditionnelle) et les stratégies d'apprentissage associées (Boulet, Savoie-Zajc et Chevrier, 1996).

24 - Elaborer des fiches de synthèse de cours (Jones, Pierce et Hunter, 1988; Saint-Onge, 2014).

25 - Identifier les causes de ses échecs pour pouvoir s'adapter (Villeneuve et al., 2010).

\subsubsection{Format des ateliers}

26 La façon dont l'étudiant assimile les connaissances doit être prise en compte lors de la mise en œuvre d'un dispositif pédagogique. Un des modes d'accompagnement que nous avons choisi est le questionnement. Lorsque celui-ci porte sur son propre fonctionnement en situation d'apprentissage, il permet une meilleure connaissance de soi et ainsi une régulation de son apprentissage mieux contrôlée, plus efficace et plus efficiente (Villeneuve et al., 2010).

27 Un des objectifs des ateliers est que l'étudiant puisse s'approprier des stratégies d'apprentissage adaptées aux exigences de la formation. Cela peut induire, chez le 
primo-entrant, un conflit cognitif difficile à surmonter, en particulier pour les étudiants en difficulté. Comme l'indique Vygotsky (1978), les pairs peuvent soutenir l'apprentissage lorsqu'il se situe dans la zone proximale de développement. Le scénario des ateliers est ainsi organisé en plusieurs temps: un premier temps de questionnement individuel, une mise en commun entre pairs par ilot (quatre étudiants), et, lorsque le thème s'y prête, une mise en commun globale. Pour finir, un apport théorique et une synthèse sont proposés. A l'instar du compagnonnage (Paul, 2009a, \$12), la mise en commun par ilot est un partage d'expérience entre pairs sur une thématique, guidé par un "tuteur ». Le rôle de ce dernier est de susciter des questionnements de la part des étudiants et de faciliter la mise en commun, l'écoute et le partage d'expérience.

\subsubsection{Planification des séances}

Les ateliers ont été programmés dès la $1^{\text {ère }}$ semaine de travaux dirigés du semestre 1 (cf. Tableau 2). L'intérêt de proposer ces séances dès la rentrée est qu'elles contribuent à l'intégration des étudiants, tant au niveau social qu'académique.

Tableau 2. Thèmes et planification des ateliers

\begin{tabular}{|l|l|l|}
\hline$N^{\circ}$ semaine & Thème de la séance & Durée \\
\hline S36 & Semaine de rentrée : cours magistraux & \\
\hline S37 & $\begin{array}{l}\text { Ma vie d'étudiant : envoyer un mail, connaître ses interlocuteurs, gérer } \\
\text { ses ressources }\end{array}$ & $45^{\prime}$ \\
\hline Les étudiants répondent à une enquête en ligne sur leurs méthodes de travail & \multicolumn{2}{|l|}{} \\
\hline S38 & Bien vivre ses études : gérer son temps & \multicolumn{2}{|l|}{} \\
\hline Les étudiants rendent un emploi du temps complété & $1 \mathrm{~h}$ \\
\hline S39 & Être efficace dans ses apprentissages (1): mémorisation et apprentissage \\
\hline S40 & Être efficace dans ses apprentissages (2) : rédiger une fiche & $45^{\prime}$ \\
\hline S42 & Contrôles mi semestre & $1 \mathrm{~h}$ \\
\hline S45 & Séance 5 - S'adapter pour mieux réussir : attribution causale & \\
\hline
\end{tabular}

\subsection{Activités pédagogiques déployées}

Les primo-entrants arrivent à l'université avec des méthodes de travail qui leur ont permis d'obtenir le bac, et d'intégrer une filière sélective (un DUT). Ils sont donc bien souvent peu enclins à changer de méthodes de travail. En conséquence, il a été mis en place au niveau de la formation, une première session d'évaluations sommatives (contrôles communs) tôt dans l'année (six semaines après la rentrée), afin de permettre aux étudiants d'avoir un retour au plus vite sur les éventuels décalages entre d'une part 
leur perception des attentes et les exigences des enseignants, et d'autre part entre leur sentiment de maîtrise et leur maîtrise effective. Il s'agit qu'ils prennent conscience des adaptations nécessaires de leurs méthodes de travail.

Chaque discipline a sa propre culture, ses propres objets d'études, ses manières de poser les problèmes et de les résoudre (Alava et Romainville, 2001). Ces cultures différentes engendrent des différences dans la manière d'aborder l'enseignement et l'apprentissage de la discipline (importance de la mémorisation, du raisonnement, de la capacité à résoudre des exercices types ...). Les difficultés cognitives peuvent être ainsi spécifiques aux disciplines. Toutefois, certaines activités pédagogiques sont reconnues pour favoriser les apprentissages (Hattie, 2012; Talbot, 2012). Les enseignants de l'équipe pédagogique impliqués dans ce projet (une petite dizaine sur 20) ont ainsi mis en place diverses activités pédagogiques.

\subsubsection{Quizz de contrôle des connaissances en début de séance de travaux dirigés}

31 Ces évaluations formatives favorisent la régulation des apprentissages et contribuent également à (Dehaene, 2012) :

32 - développer sa capacité d'attention en identifiant les connaissances importantes du cours;

33 - consolider ses acquis, en réactivant régulièrement les connaissances;

34 - avoir un feedback régulier sur ce qui est maitrisé ou pas;

35 - maintenir la motivation et l'engagement, en renforçant le sentiment de compétence et de contrôlabilité sur sa réussite.

\subsubsection{Exercices travaillés (explicitation par l'expert)}

Les manières de poser les problèmes et de les résoudre, propres à chaque discipline, sont souvent implicites. Le principe de l'exercice travaillé est que l'enseignant guide l'étudiant vers l'autonomie, en trois phases (Gauthier, 2013) :

1. modelage : l'enseignant résout un exercice type en explicitant sa démarche, ses questionnements, le choix des éléments pertinents du cours pour résoudre le problème;

2. entraînement en séance de travaux dirigés : les étudiants résolvent un exercice avec un feedback individualisé ou par binôme de la part de l'enseignant;

3. vérification des acquis en autonomie : proposition d'une liste d'exercices, en ligne ou pas, et des corrigés associés.

\subsubsection{Elaboration de fiches de synthèse de cours}

Comme l'indique Tardif (1992), «l'enseignant stratégique vise à fournir à l'élève les moyens de devenir autonome dans ses démarches d'apprentissage et de traitement des informations... » (p. 298). Ainsi, dans les disciplines qui s'y prêtent, les étudiants sont accompagnés pour construire des fiches de synthèse de cours : l'enseignant explicite le type des connaissances (déclaratives, procédurales ou conditionnelles) et les stratégies d'apprentissage les plus appropriées. 


\section{4. Évaluer le dispositif d'accompagnement}

41 Notre démarche vise à favoriser la transition lycée-université. Nous avons donc choisi d'évaluer le dispositif proposé d'une part au regard des résultats académiques au semestre 1 (validation/échec, abandon), et d'autre part au regard de la perception de maîtrise des étudiants sur les thèmes abordés dans les ateliers (Bournaud et Pamphile, 2020).

\subsection{Impact sur les résultats académiques au semestre 1}

L'IUT de Sceaux comporte deux départements Gestion des Entreprises et des Administrations (GEA) dans lesquels les primo-entrants sont répartis de manière à avoir la même répartition dans les deux départements de filles/garçons et types et séries de Bac (Bac généraux : série $\mathrm{S}$ - Scientifique, série ES -Economique et Social, Bac technologique série STMG - Sciences et Technologies du Management et de la Gestion). Nous nommons "groupe test " le département dans lequel le dispositif a été mis en place, et "groupe contrôle " l'autre; leurs effectifs sont respectivement 179 et 185 étudiants.

Le Tableau 3 ci-dessous présente les résultats académiques au semestre 1: taux de validation et taux d'abandon, pour le groupe test et le groupe contrôle.

Tableau 3. Résultats au semestre 1 en 2018/2019

\begin{tabular}{|l|l|l|l|l|}
\hline & \multicolumn{2}{|l|}{$\%$ Validation S1 } & \multicolumn{2}{l|}{$\%$ Abandons } \\
\hline & tous bacs & bac STMG & tous bacs & bac STMG \\
\hline Groupe test & $74,7 \%$ & $57,7 \%$ & $2,2 \%$ & $3,4 \%$ \\
\hline Groupe contrôle & $71,4 \%$ & $48,5 \%$ & $8,1 \%$ & $14,7 \%$ \\
\hline
\end{tabular}

Concernant le taux de validation du S1, on ne constate pas de différence significative entre le groupe test et le groupe contrôle, y compris pour les bacs technologiques (STMG) (Test de Khi2, p-value >0,1). Notons que les bacs généraux (séries $\mathrm{S}$ et ES) n'ont pas été distingués car il n'y a pas de différence significative de résultats au semestre 1 entre les étudiants de ces deux séries de bac.

En termes de taux d'abandon, on constate dans le groupe test un taux d'abandon très faible en 2018/2019 (2,2 \% pour le groupe test contre 8,1 \% pour le groupe de contrôle). La remarque est la même si l'on ne regarde que les bacs SMTG (3,4 \% pour le groupe test, contre $14,7 \%$ pour le groupe contrôle). Ces différences sont significatives (Test de Khi2, p-values $<0,05$ ).

Donc si les résultats ne permettent pas de conclure que le dispositif a eu un impact sur la validation du semestre 1 , en revanche, ils permettent de conclure qu'il a un impact sur la persistance des étudiants dans la formation. 


\subsection{Impact des ateliers sur la perception de maîtrise}

47 Le choix méthodologique que nous avons fait est d'évaluer les ateliers à partir d'une auto-évaluation par les étudiants de leur perception de maîtrise, avant et après les ateliers (Gérard, 2003).

\subsubsection{Questionnaire}

Nous avons demandé aux étudiants d'estimer leur niveau de maîtrise avant et après l'atelier au regard de diverses thèmes abordés dans les ateliers. Ainsi, des propositions ont été formulées et soumises aux étudiants, avec une échelle mixte de perception de maîtrise avant et après le dispositif (voir Tableau 4 ci-dessous). Ces propositions sont détaillées dans la suite.

Tableau 4. Exemple de proposition

\begin{tabular}{|l|l|l|l|l|l|l|l|l|l|l|l|l|}
\hline Q1-Je connais les personnes ressources et leur rôle dans ma formation \\
\hline $\begin{array}{l}-5 \text { Non, pas du } \\
\text { tout }\end{array}$ & -4 & -3 & -2 & $\begin{array}{l}-1 \quad \text { Non, pas } \\
\text { vraiment }\end{array}$ & $\begin{array}{l}1 \\
\text { plutôt }\end{array}$ & 2 & 3 & 4 & $\begin{array}{l}5 \text { Oui, tout à } \\
\text { fait }\end{array}$ \\
\hline Avant & & & & & & & & & & \\
\hline Après & & & & & & & & & & \\
\end{tabular}

49 Sur les 179 étudiants de la promotion du groupe test, 133 ont répondu à cette enquête d'évaluation des ateliers, qui a été administrée juste après le dernier atelier. Rappelons que toutes les séances des ateliers étaient obligatoires pour tous les étudiants du groupe test. Nous avons vérifié que l'échantillon est représentatif en termes de répartition validation/échec au semestre 1 (Test du khi-2: p-value $<0,05$ ) et en termes de répartition des bacs (ES, S, STMG) (Test du khi-2: $p$-value $<0,05)$.

\subsubsection{Méthodologie d'analyse des réponses}

- Pour chacune des propositions, avant l'atelier et après l'atelier, on calcule :

Image
\begin{tabular}{|l|l|l|}
\hline Moyenne & $\bar{x}$ & 1000814400003E83000012002AAE6D1A393C2AEC.emf \\
\hline $\begin{array}{l}\text { Coefficient } \\
\text { de variation }\end{array}$ & $\frac{\sigma}{\bar{x}}$ & $\begin{array}{l}\text { Ecart-type des réponses. Noté en pourcentage, c'est un } \\
\text { indicateur d'hétérogénéité des réponses. }\end{array}$ \\
\hline
\end{tabular}

51 - Par ailleurs, on calcule pour chacune des propositions : 
Image 1000C1A000003E8300001907B42AEB604D75DEAE.emf

\begin{tabular}{|l|c}
\hline $\begin{array}{l}\text { Gain brut } \\
\text { moyen }\end{array}$ & $\overline{\boldsymbol{x}_{\text {après }}-\boldsymbol{x}_{\text {avant }}}$ \\
\hline Gain relatif & $\bar{\Delta}=\left(\frac{\overline{\boldsymbol{x}_{\text {après }}-\boldsymbol{x}_{\text {avant }}}}{\overline{\boldsymbol{x}_{\text {max }}-\boldsymbol{x}_{\text {avant }}}}\right)$ \\
\hline
\end{tabular}

Correspond à ce qui a été «gagné » lors de l'atelier.

Correspond à ce qui aurait pu être gagné lors de l'atelier ( $\boldsymbol{x}_{\max }$ étant la valeur maximale à atteindre).

On considérera qu'un atelier a permis un apprentissage si le gain relatif est supérieur à $30 \%$ (Gérard, 2003).

Illustrons les gains, brut et relatif, en considérant l'exemple du tableau 5 ci-dessous de quatre groupes d'étudiants.

Tableau 5. Illustration du gain brut et du gain relatif

\begin{tabular}{|l|l|l|l|l|}
\hline Groupe & $\boldsymbol{x}_{\text {avant }}$ & $\boldsymbol{x}_{\text {après }}$ & Gain brut & Gain relatif \\
\hline 1 & -5 & -4 & 1 & $10 \%$ \\
\hline 2 & 4 & 5 & 1 & $100 \%$ \\
\hline 3 & -1 & 1 & 2 & $30 \%$ \\
\hline 4 & -4 & 1 & 5 & $56 \%$ \\
\hline
\end{tabular}

On voit sur cet exemple que le gain brut seul n'est pas un bon indicateur de l'effet positif d'un atelier: les groupes 1 et 2 ont le même gain brut, mais pas le même gain relatif; les groupes 3 et 4 ont atteint le même niveau après l'atelier, mais ils n'ont pas le même gain relatif (ils ne partaient pas du même niveau avant l'atelier).

\subsubsection{Analyse des réponses par atelier}

Dans ce qui suit, nous présentons, par atelier, l'analyse des réponses au questionnaire sur la perception de maîtrise.

Tableau 6. Atelier 1 - personnes ressources

\begin{tabular}{|c|c|c|c|c|}
\hline ATELIER 1 - PERSONNES RESSOURCES & & Moyenne & $\begin{array}{l}\text { Coefficient de } \\
\text { variation }\end{array}$ & $\begin{array}{l}\text { Gain } \\
\text { relatif }\end{array}$ \\
\hline \multirow{2}{*}{$\begin{array}{l}\text { Q1-Je connais les personnes ressources et leur rôle dans } \\
\text { ma formation (responsable amphi, chargé de TD, } \\
\text { délégué de TD, secrétariat, direction des études, chef de } \\
\text { département, ...) }\end{array}$} & Avant & -1 & $245,00 \%$ & \multirow[b]{2}{*}{$46,0 \%$} \\
\hline & Après & 1,8 & $95,90 \%$ & \\
\hline Q2-Je sais rédiger un mail professionnel pour & Avant & 1,2 & $183,70 \%$ & \\
\hline
\end{tabular}

m'adresser à une personne ressource? 
Q1 - Les étudiants, répondent plutôt négativement avant l'atelier, mais, plutôt positivement après. L'amélioration est faible (la moyenne passe de -1 à 1,8 ), mais significative $(p$-value $<0,001)$. Par ailleurs, après les ateliers les réponses sont moins hétérogènes (le coefficient de variation a diminué). Enfin, on peut considérer que l'atelier a eu un effet positif (gain relatif supérieur à $30 \%$ ).

Q2 - L'amélioration est importante, et significative. Par ailleurs, après les ateliers les réponses sont moins hétérogènes. Enfin, on peut considérer que l'atelier a eu un effet positif (gain relatif supérieur à $30 \%$ ).

Tableau 7. Atelier 2 - Bien vivre ses études, gérer son temps

\begin{tabular}{|l|l|l|l|l|}
\hline $\begin{array}{l}\text { ATELIER 2 - BIEN VIVRE SES ETUDES, GERER SON } \\
\text { TEMPS }\end{array}$ & Moyenne & $\begin{array}{l}\text { Coefficient de } \\
\text { variation }\end{array}$ & $\begin{array}{l}\text { Gain } \\
\text { relatif }\end{array}$ \\
\hline $\begin{array}{l}\text { Q3-J'ai conscience des différences entre le lycée et l'IUT } \\
\text { en termes de temps de transport, d'organisation des } \\
\text { enseignements, nombre d'heures de travail, ... }\end{array}$ & Avant & 1,5 & $127,40 \%$ & \multirow{2}{*}{$63,5 \%$} \\
\cline { 2 - 5 } & Avrès & 3,6 & $46,60 \%$ & \multirow{2}{*}{$50,3 \%$} \\
\cline { 2 - 5 } Q4-J'ai analysé mon emploi du temps actuel & Après & 2,9 & $58,50 \%$ & \\
\hline \multirow{2}{*}{ Q5-Je planifie mon travail chaque semaine } & Avant & $-0,2$ & $1496,20 \%$ & \multirow{2}{*}{$31,5 \%$} \\
\cline { 2 - 5 } & Après & 1,4 & $120,90 \%$ & \\
\hline
\end{tabular}

Q3 et Q4 - L'amélioration est importante, et significative. Par ailleurs après les ateliers les réponses sont moins hétérogènes. Enfin, on peut considérer que l'atelier a eu un effet positif sur ces deux volets (gains relatifs supérieurs à $30 \%$ ).

Q5 - Les étudiants répondent plutôt négativement avant les ateliers, mais positivement après les ateliers. L'amélioration est faible, mais significative. Sur le thème de la gestion du temps, abordée dans l'atelier 2 , on peut voir que l'hétérogénéité des réponses des étudiants a été notablement réduite, pour les trois propositions Q3, Q4 et Q5.

Toutefois, concernant la planification hebdomadaire du travail (Q5), même si l'hétérogénéité a très largement diminué, le gain relatif est faible (proche de $30 \%$ ), l'effet de l'atelier est donc limité. 
Figure 1. Ateliers 3 et 4 - Etre efficace dans ses apprentissages

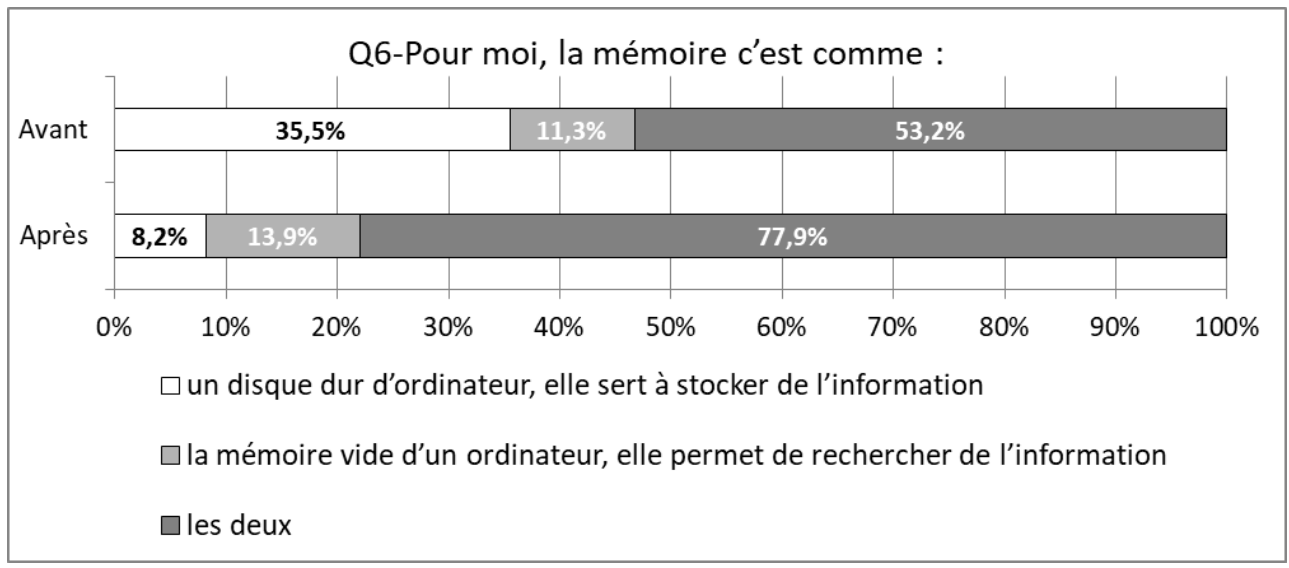

61 La différence de représentation de la mémoire par les étudiants, avant/après atelier est significative (Test du Khi-deux : $p$-value <0,001). Il apparaît clairement que l'atelier 3 qui abordait la mémorisation a fait changer la représentation des étudiants, ce qui était l'objectif visé.

Tableau 8. Ateliers 3 et 4 - Etre efficace dans ses apprentissages

\begin{tabular}{|c|c|c|c|c|}
\hline $\begin{array}{l}\text { ATELIERS } 3 \text { et } 4 \text { - ÊTRE EFFICACE DANS SES } \\
\text { APPRENTISSAGES }\end{array}$ & & Moyenne & $\begin{array}{l}\text { Coefficient de } \\
\text { variation }\end{array}$ & $\begin{array}{l}\text { Gain } \\
\text { relatif }\end{array}$ \\
\hline \multirow{2}{*}{$\begin{array}{l}\text { Q7-J'ai analysé mes habitudes pour préparer un } \\
\text { contrôle }\end{array}$} & Avant & $-0,1$ & $1743,60 \%$ & \multirow{2}{*}{$61,3 \%$} \\
\hline & Après & 1,8 & $96,40 \%$ & \\
\hline \multirow{2}{*}{$\begin{array}{l}\text { Q8-Quand j'ai une matière à étudier, je prends le } \\
\text { temps d'identifier les notions importantes du cours }\end{array}$} & Avant & 1,3 & $182,30 \%$ & \multirow{2}{*}{$25,0 \%$} \\
\hline & Après & 2,6 & $66,10 \%$ & \\
\hline \multirow{2}{*}{$\begin{array}{l}\text { Q9-Quand j'ai une matière à étudier, je sais identifier } \\
\text { les connaissances déclaratives, procédurales et } \\
\text { conditionnelles }\end{array}$} & Avant & $-0,8$ & $319,70 \%$ & \multirow{2}{*}{$38,1 \%$} \\
\hline & Après & 0,5 & $358,80 \%$ & \\
\hline \multirow{2}{*}{$\begin{array}{l}\text { Q10-Quand j'ai une matière à étudier, j'utilise des } \\
\text { fiches pour apprendre }\end{array}$} & Avant & 0,2 & $1469,30 \%$ & \multirow{2}{*}{$33,5 \%$} \\
\hline & Après & 1,5 & $112,90 \%$ & \\
\hline
\end{tabular}

Q7 à Q10 - Pour ces quatre propositions, l'amélioration est faible, voire très faible pour Q9, mais est significative.

63 Les coefficients de variation de Q7, Q8 et Q10 montrent que les réponses à ces propositions après les ateliers sont moins hétérogènes, avec une variation très importante concernant l'analyse de ses habitudes pour préparer un contrôle (Q7) et l'utilisation de fiches pour apprendre (Q10). En revanche, concernant l'identification des connaissances déclaratives, procédures et conditionnelles (Q9), l'hétérogénéité n'a pas diminué après les ateliers, et reste très élevée. 
On peut considérer qu'il y a un effet positif d'apprentissage pour Q7, Q9 et Q10 (gain relatif supérieur à $30 \%$ ), notamment Q7; en revanche ce n'est pas le cas pour Q8 (Quand j'ai une matière à étudier, je prends le temps d'identifier les notions importantes du cours) dont le gain relatif est de $25 \%$.

Figure 2. La révision des contrôles

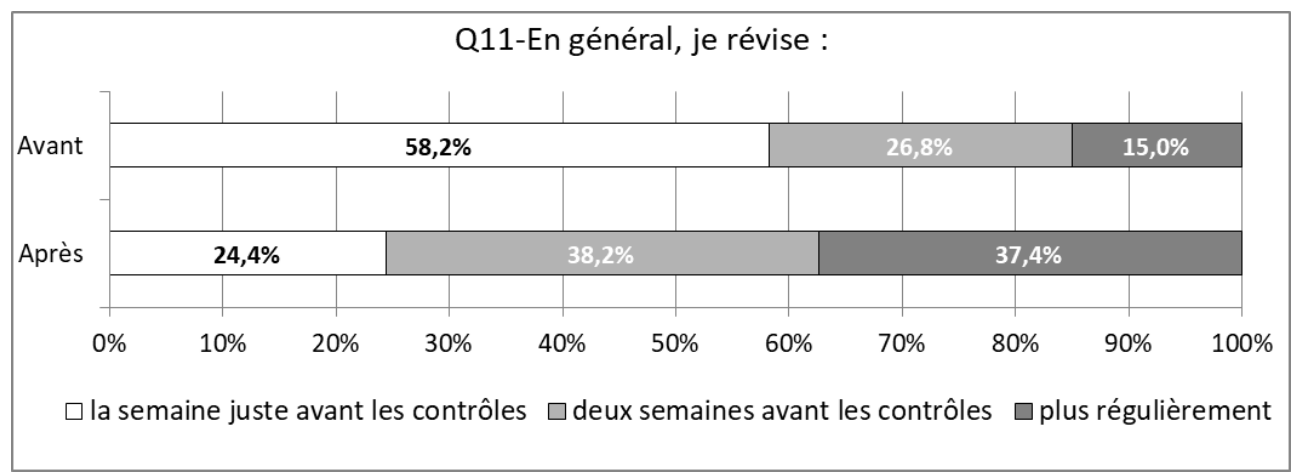

La différence concernant la révision des contrôles avant/après est significative (Test du Khi-deux $p$-value $<0,001)$. Les ateliers ont eu pour effet de faire changer les pratiques déclarées pour la révision des contrôles. On notera cependant l'existence d'un «frein »: $24,4 \%$ des étudiants ont préparé les contrôles de mi semestre la semaine précédant les contrôles.

Tableau 9. Atelier 5 - S'adapter pour mieux réussir

\begin{tabular}{|l|l|l|l|l|}
\hline ATELIER 5 - S'ADAPTER POUR MIEUX REUSSIR & & Moyenne & $\begin{array}{l}\text { Coefficient de } \\
\text { variation }\end{array}$ & $\begin{array}{l}\text { Gain } \\
\text { relatif }\end{array}$ \\
\hline \multirow{2}{*}{$\begin{array}{l}\text { Q12-En général avant un contrôle, je sais quoi faire } \\
\text { pour avoir une bonne note }\end{array}$} & Avant & 1,3 & $131,70 \%$ & \multirow{2}{*}{$23,9 \%$} \\
\cline { 2 - 5 } & Après & 2,3 & $72,90 \%$ & \multirow{2}{*}{$34,2 \%$} \\
\hline $\begin{array}{l}\text { Q13-Quand j'ai une mauvaise note, ou moins bonne } \\
\text { que celle que j'attendais, je prends le temps d'en } \\
\text { identifier les causes }\end{array}$ & Avant & 0,7 & $333,30 \%$ & \\
\cline { 2 - 5 } & Après & 2,1 & $80,70 \%$ & \\
\hline
\end{tabular}

Q12 et Q13 - L'amélioration est peu importante, mais significative. Par ailleurs après les ateliers les réponses sont moins hétérogènes, notamment concernant l'attribution causale (Q13). On ne peut pas considérer qu'il y a eu un effet d'apprentissage concernant savoir quoi faire pour avoir une bonne note au contrôle (Q12), et cet effet est léger concernant l'attribution causale (gain relatif de $34,2 \%$ ). 
Figure 3. Q14 - Pour conclure (sur l'utilité des ateliers)

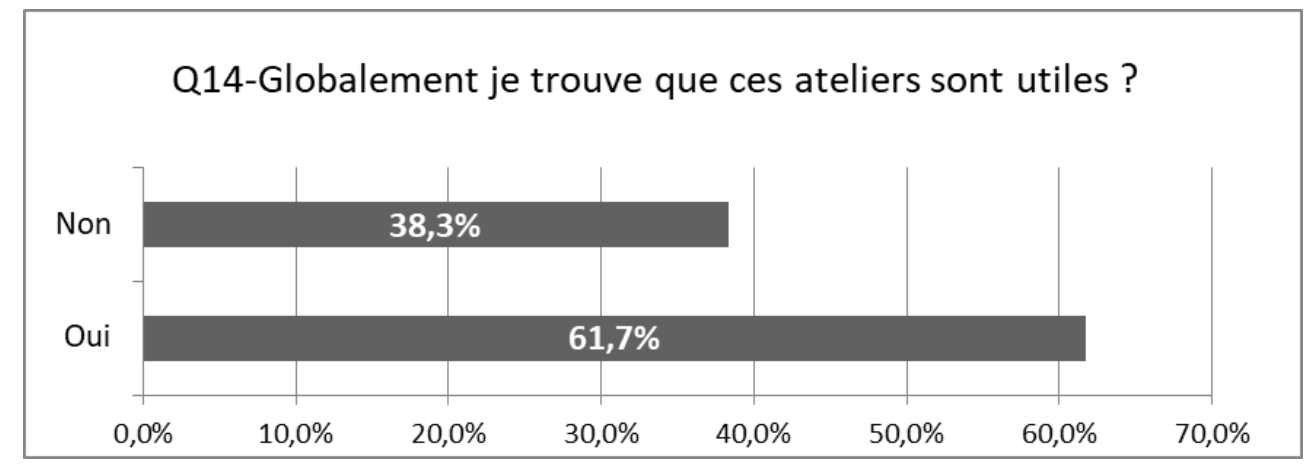

\subsubsection{Analyse des réponses par Bac}

Pour affiner notre analyse, nous avons comparé les comportements des différents bacs (ES, S ou STMG) sachant que les acquis et méthodes ne sont pas les mêmes selon le Bac préparé.

Perception de maîtrise avant/après

Nous avons calculé la perception avant/après atelier selon le Bac pour toutes les propositions. Nous présentons dans le Tableau 10 uniquement celles pour lesquelles les différences entre Bac avant les ateliers sont significatives (Test non paramétrique des rangs signés, $p$-value $<0,05$ ).

Tableau 10. Propositions pour lesquelles la perception de maîtrise est significativement différente selon le Bac

\begin{tabular}{|c|c|c|c|c|c|}
\hline & \multicolumn{4}{|c|}{ 1001972C00003E830000215EA93498FFC3С2FBC2.em } \\
\hline \multirow{2}{*}{\multicolumn{2}{|c|}{ Image }} & ES & $S$ & STMG & \\
\hline & & $\overline{\boldsymbol{x}}$ & $\overline{\boldsymbol{x}}$ & $\overline{\boldsymbol{x}}$ & \\
\hline \multirow{2}{*}{$\begin{array}{l}\text { Q4-J'ai analysé mon } \\
\text { emploi du temps actuel? }\end{array}$} & Avant & 1,1 & 2,8 & 0,7 & $\begin{array}{l}\text { Différence significative } \\
(p \text {-value }=0,05)\end{array}$ \\
\hline & Après & 2,7 & 4,3 & 2,9 & $\begin{array}{l}\text { Pas de différence significative } \\
(p \text {-value }=0,13)\end{array}$ \\
\hline \multirow{2}{*}{$\begin{array}{l}\text { Q12- En général avant } \\
\text { un contrôle, je sais quoi } \\
\text { faire pour avoir une } \\
\text { bonne note? }\end{array}$} & Avant & 1,4 & 3,0 & 1,0 & $\begin{array}{l}\text { Différence significative ( } p \text { - } \\
\text { value }=0,02 \text { ) }\end{array}$ \\
\hline & Après & 2,3 & 3,2 & 2,2 & $\begin{array}{l}\text { Pas de différence significative } \\
(p \text {-value }=0,33)\end{array}$ \\
\hline
\end{tabular}

On constate ainsi qu'il n'y a plus de différence significative de perception entre les Bac après les ateliers. On peut penser que les ateliers ont homogénéisé les réponses des étudiants de bac différent concernant l'analyse de son emploi du temps (Q4) et savoir quoi faire pour avoir une bonne note (Q12).

\section{Gain relatif}


72 Nous avons analysé le gain relatif selon le Bac pour toutes les propositions. Nous présentons dans le Tableau 11 uniquement celle pour laquelle les différences entre Bacs sont significatives (Test non paramétrique des rangs signés, $p$-value $<0,05$ ).

Tableau 11. Proposition pour laquelle le gain relatif est significativement différent selon les Bacs

\begin{tabular}{|l|l|l|l|l|}
\hline \multicolumn{6}{|l|}{ Q7-J'ai analysé mes habitudes pour préparer un contrôle } \\
\hline & ES & S & STMG & \\
\hline $\begin{array}{l}\text { Gain relatif } \\
\text { moyen }\end{array}$ & $47,2 \%$ & $58,3 \%$ & $\mathbf{8 4 , 5} \%$ & Différence significative $(p$-value $=0,05)$ \\
\hline
\end{tabular}

73 Il ressort de ce tableau que les ateliers ont un apport positif pour tous les Bac (gain relatif $>30 \%$ ), et en particulier pour les Bac STMG, concernant l'analyse de ses habitudes de préparation d'un contrôle.

\section{Discussion}

74 Si rien ne prouve que le dispositif mis en place a eu un impact sur la validation du semestre 1 , on peut conclure qu'il a réduit de manière significative le taux d'abandons/ démissions, y compris chez les bacs technologiques (STMG), population éprouvant plus de difficulté dans la transition lycée-université. D'autres travaux ont montré que l'introduction d'actions d'aide obligatoires a pour effet que les plus faibles tendent à moins abandonner (Perret et Berthaud, 2015). Par ailleurs, le sentiment de maitrise des étudiants a significativement augmenté après les ateliers, en particulier chez les bacs technologiques. Différents travaux confirment le fait qu'un étudiant qui se sent affilié, accompagné et qui a un sentiment d'efficacité personnelle positif persiste dans sa formation (Michalot, Seguy et Bouladon, 2018; Neuville, Frenay, Noel et Wertz, 2013). Enfin, on constate que le dispositif a réduit l'hétérogénéité initiale entre les étudiants en termes de maitrise. Talbot (2012) indique à ce propos dans sa méta-analyse des pratiques enseignantes, que « les pratiques d'enseignement efficaces sont également les pratiques qui réduisent les écarts de performances entre les élèves forts et les élèves faibles ».

L'évaluation des ateliers nous permet d'identifier des points d'amélioration :

76 - le gain relatif n'est pas significatif pour les questions Q8 (Quand j'ai une matière à étudier, je prends le temps d'identifier les notions importantes du cours) et Q12 (En général avant un contrôle je sais quoi faire pour avoir une bonne note). Un travail de l'équipe pédagogique sur l'explicitation d'une part des objectifs d'apprentissage visés et des critères d'évaluation, et d'autre part sur les notions importantes du cours peut être envisagé;

77 - le gain relatif est peu important pour Q9 (Quand j'ai une matière à étudier, je sais identifier les connaissances déclaratives, procédurales et conditionnelles) et l'hétérogénéité entre les étudiants reste très élevée après l'atelier. Là encore un travail de l'équipe pédagogique sur les différents types de connaissances et l'accompagnement des étudiants dans la construction de fiches, peut être envisagé; 
78 préoccupation importante des universités. L'efficacité des dispositifs d'accompagnement de type méthodologie est limitée si les démarches proposées ne sont pas contextualisées. Par ailleurs, le soutien disciplinaire ne suffit pas pour des étudiants n'ayant pas de méthodes d'étude adaptées au contexte universitaire. Nous avons élaboré et mis en œuvre un accompagnement des primo-entrants visant à faciliter la transition lycée-université qui repose d'une part sur des ateliers et d'autre part sur des activités pédagogiques déployées dans les enseignements disciplinaires.

81 L'efficacité du dispositif a été évaluée en comparant les résultats académiques au semestre 1 (taux de validation/échec et taux d'abandon) d'un groupe test et d'un groupe contrôle. Par ailleurs, une enquête a été menée sur la perception de maitrise des étudiants des thèmes abordés dans les ateliers, avant et après ceux-ci. Si rien ne prouve que le dispositif a eu un impact significatif sur le taux de validation du S1, on constate qu'il a réduit de manière significative le taux d'abandon, et également l'hétérogénéité des étudiants en matière de perception de maîtrise après les ateliers. Des améliorations sont toutefois envisagées, d'une part sur les activités pédagogiques déployées dans les enseignements (notamment pour identifier les points importants d'un cours, et le type de connaissances travaillées), et d'autre part sur les ateliers (distinguer les trois types des connaissances, élaborer des fiches de synthèse, planifier son travail). La réflexion sur l'articulation entre les ateliers et les enseignements disciplinaires doit être approfondie. Nous envisageons notamment d'illustrer certains ateliers (types de connaissances, fiche de synthèse) sur différentes disciplines du semestre en sollicitant les collègues afin de disposer de contenus appropriés, et que ceux-ci soient retravaillés sous la forme vue en atelier dans les séances de travaux dirigés disciplinaires. Par ailleurs, il s'agit de poursuivre l'accompagnement dans les enseignements disciplinaires au semestre 2 afin que les étudiants s'approprient les méthodes sur lesquelles ils ont réfléchi pendant les ateliers. 


\section{BIBLIOGRAPHY}

Alava, S. et Romainville, M. (2001). Les pratiques d'étude, entre socialisation et cognition. Revue française de pédagogie, 1(136), 159-180.

Bernard, H. (2011). Comment évaluer, améliorer, valoriser l'enseignement supérieur? Guide pratique à l'usage des professeurs, cadres dirigeants, responsables pédagogiques. De Boeck Supérieur.

Berthaud, J. (2017). L'intégration sociale étudiante : relations et effets au sein des parcours de réussite en Licence [these de doctorat, Université Bourgogne Franche-Comté].

Biggs, J. B. (2011). Teaching for quality learning at university: What the student does. McGraw-hill education.

Boulet, A., Savoie-Zajc, L. et Chevrier, J. (1996). Les stratégies d'apprentissage à l'université (Vol. 6). Presses de l'Université du Quebec.

Bournaud, I., Depoutot V. et Pamphile P. (2018, 10-12 janvier), Une étude croisée pour identifier les facteurs d'échec/réussite au semestre 1 afin d'aider les étudiants dans leurs apprentissages. Actes du $30^{e}$ colloque de l'ADMEE. Campus Belval, Esch-sur-Alzette, Luxembourg. https:// orbilu.uni.lu/bitstream/10993/35653/1/ADMEE_2018_Programme_colloque_web_2.pdf Bournaud I. et Pamphile P. (2020, janvier), Quels critères considérer pour évaluer un dispositif d'aide aux primo-entrants à l'université? Le cas d'ateliers de méthodologie, Symposium au $32^{e}$ colloque de l'ADMEE. Casablanca, Maroc.

Cosnefroy, L., Hoffmann, C. et Douady, J. (2014). L'accompagnement méthodologique. Recherche \& formation, 77(3), 29-44.

Dehaene, S. (2012, 20 novembre). Les grands principes de l'apprentissage [vidéo]. Collège de France. https://www.college-de-france.fr/site/stanislas-dehaene/symposium-2012-11-20-10h00.htm

Dupont, S., De Clercq M. et Galand. B. (2015). Les prédicteurs de la réussite dans l'enseignement supérieur. Revue française de pédagogie, 2, 105-136.

Gauthier, C., Bissonnette S. et Richard, M. (2013). Enseignement explicite et réussite des élèves. La gestion des apprentissages. ERPI.

Gérard, F. M. (2003). L'évaluation de l'efficacité d'une formation. Gestion 2000, 20(3), 13-33.

Hattie, J. (2012). Visible Learning for Teachers. Maximizing Impact on Learning. Routledge.

Houart, M. (2017). Réussir sa première année ( $2^{\mathrm{e}}$ éd.). De Boeck Supérieur.

Houdé, O. (2011). Imagerie cérébrale, cognition et pédagogie-Imagerie et cognition (6). Médecine/ sciences, 27(5), 535-539.

Jones, B. F., Pierce, J. et Hunter, B. (1988). Teaching students to construct graphic representations. Educational Leadership, 46(4), 20-25.

Lison, C., Bédard, D., Beaucher, C. et Trudelle, D. (2014). De l'innovation à un modèle de dynamique innovationnelle en enseignement supérieur. Ripes, 30(1), 1-20. https://doi.org/ 10.4000/ripes.771

Lison, C. et Jutras, F. (2014). Innover à l'université : penser les situations d'enseignement pour soutenir l'apprentissage. Ripes, 30(1), 1-9. https://doi.org/10.4000/ripes.769 
Michalot, T., Seguy, J. et Bouladon, A. (2018). Sentiment de légitimité : un facteur à prendre en compte pour persévérer et réussir à l'université [communication]. Colloque international : Apprendre, Transmettre, Innover à et par l'Université Saison_2. https://hal.archives-ouvertes.fr/ hal-01939041/document

Michaut, C. (2003). L'efficacité des dispositifs d'aide aux étudiants dans les universités. Recherche et formation.

Michaut, C. et Romainville, M. (2012). Réussite, échec et abandon dans l'enseignement supérieur. De Boeck.

Neuville, S., Frenay, M., Noel, B. et Wertz, V. (2013). Persévérer et réussir à l'université. Presses universitaires de Louvain.

Noël, B. et Romainville, M. (1998). Accompagner les étudiants. Dans L'étudiant-apprenant. Grille de lecture pour l'enseignement universitaire. De Boeck.

Paivandi, S. (2015). Apprendre à l'université. De Boeck supérieur. Pédagogies en développement.

Paul, M. (2009a). Autour du mot Accompagnement. Recherche et formation, (62), 91-108.

Paul, M. (2009b). L'accompagnement dans le champ professionnel. Savoirs, (2), 11-63.

Perret, C. (2014). Retour d'expérience d'une première évaluation du Plan Réussite en Licence à l'Université de Bourgogne : les leçons d'un difficile recueil des points de vue enseignants et étudiants. Ripes, 30(2), 1-18. https://doi.org/10.4000/ripes.816

Perret, C. (2015). Le Plan Réussite en Licence. Éditions Universitaires de Dijon.

Perret, C. et Berthaud, J. (2015). Quels effets sur la réussite étudiante de dispositifs d'aide et de soutien obligatoires? Dans Le Plan Réussite en Licence (p. 169-197). Éditions Universitaires de Dijon.

Perret C. et Morlaix S. (2015). Quels nouveaux regards sur la sélection des étudiants à l'université via le plan Réussite en Licence? Dans Le Plan Réussite en Licence (p. 143-167). Éditions Universitaires de Dijon.

Philion, R., Bourassa, M., Leblanc, R., Plouffe, D. et Arcand, I. (2010). Persistance et réussite aux études postsecondaires : étude d'un accompagnement personnalisé pour étudiants en situation d'échec. Revue de recherche appliquée sur l'apprentissage, 3(6), 1-27.

Raucent, B., Verzat, C. et Villeneuve, L. (dir.) (2010). Accompagner des étudiants : Quels rôles pour l'enseignant? Quels dispositifs? Quelles mises en cuvre? De Boeck Supérieur.

Reverdy, C. (2017). L'accompagnement à l'école : dispositifs et réussite des élèves. Dossier de veille de l'IFÉ, (119).

Romainville, M. (2000). Savoir comment apprendre suffit-il à mieux apprendre? Pour une pensée réflexive en éducation, 71-86.

Saint-Onge, M. (2014). Moi j'enseigne, mais eux apprennent-ils? (5 éd.). Beauchemin; Chronique sociale.

Salmon, D., Baillet, D., Boulvain, M., Cobut, B., Coupremanne, M., Duchâteau, D., Lanotte, A.-F., Dubois, P., Goemaere, S., Noël, B., Houart, M. et Slosse, P. (2009). Construction d'un outil d'évaluation de la qualité des actions d'accompagnement pédagogique. Synthèse d'échanges et d'analyse de pratiques professionnelles en Communauté française de Belgique. Ripes, 25(2), 1-19. https://doi.org/10.4000/ripes.252 
Sanchez, É. et Monod-Ansaldi, R. (2015). Recherche collaborative orientée par la conception. Un paradigme méthodologique pour prendre en compte la complexité des situations d'enseignement-apprentissage. Éducation et didactique, 9(2), 73-94.

Talbot, L. (2012). Les recherches sur les pratiques enseignantes efficaces. Synthèse, limites et perspectives. Questions vives. Recherches en éducation, 6(18), 129-140.

Tardif, J. (1992). L'enseignement stratégique. Éditions Logiques.

Verzat, C. (2010). Pourquoi parler d'accompagnement des étudiants aujourd'hui? Dans B.

Raucent, C. Verzat, et L. Villeneuve (dir.), Accompagner des étudiants. Quels rôles pour l'enseignant? Quels dispositifs? Quelles mises en œuvre? (p. 27-50). De Boeck Supérieur.

Villeneuve, L., Leblanc J., Ruph F. et Lemieux, L. (2010). Le questionnement et la réflexivité. Dans B. Raucent, C. Verzat et L. Villeneuve (dir.), Accompagner des étudiants : Quels rôles pour l'enseignant? Quels dispositifs? Quelles mises en œuvre? (p. 158-185). De Boeck Supérieur.

Vygotsky, L. S. (1978). Mind in society. Harvard University Press.

\section{NOTES}

1. http://www.enseignementsup-recherche.gouv.fr/cid20651/plan-pour-la-reussite-enlicence-730-millions-d-euros-d-ici-2012.html

2. Rapport du Comité de suivi de la licence et de la licence professionnelle. Année 2010-2011. (2011). CPU/Paris. http://www.cpu.fr/wp-content/uploads/2013/07/rapport_CSL_2010_2011.pdf 3. GEA est une des 24 spécialités de DUT (https://www.iut.fr/formations-et-diplomes/lesspecialites/les-specialites-de-dut.html)

\section{ABSTRACTS}

Accompanying first year students in the transition from high school to university is now a major concern for universities. This article presents a support program designed for first-year students at university. The program is based first on workshops whose aim is to help students question their working methods in order to fit academic requirements. The workshops focus on time management, learning effectively, identifying causes for failure or success. All the first-year students attend the workshops.

Besides the program also relies on teaching methods which are shared by the teaching staff, whether in structuring projects or in classroom practice.

The program has been assessed for its impact on the one hand on the students' academic success in semester 1 (fail/pass, dropout rates), and on the other hand on whether the students felt more proficient in the skills taught in the workshops.

The program does not appear to impact student success. However, the dropout rate has significantly decreased. Besides after following the workshops, many more students expressed a sense of competence regarding working methods at university. 
Accompagner les primo-entrants dans la transition lycée-université est aujourd'hui une préoccupation importante des universités. Cet article présente un dispositif d'accompagnement de primo-entrants dans une formation universitaire. Ce dispositif repose, d'une part sur des ateliers visant à faire réfléchir les primo-entrants sur leurs méthodes de travail au regard des exigences universitaires, d'autre part sur diverses activités pédagogiques qui sont déployées dans les enseignements disciplinaires. Le dispositif a été évalué au regard des résultats académiques au semestre 1 (validation/échec, abandon) et de l'évolution de la perception de maîtrise des étudiants sur les thèmes abordés dans les ateliers. Si les résultats ne permettent pas de dire que le dispositif a eu un impact sur les résultats académiques au semestre 1 , en revanche le taux d'abandon a été significativement réduit. Par ailleurs, les ateliers ont permis de réduire l'hétérogénéité entre les étudiants concernant leur perception de maîtrise de méthodes de travail adaptées au contexte universitaire.

INDEX

Mots-clés: transition lycée/université, accompagnement, méthodes de travail, évaluation d'un dispositif

\section{AUTHORS}

\section{ISABELLE BOURNAUD}

Didasco - EST, EA1610, UFR Sciences, Université Paris Sud, Université Paris Saclay, isabelle.bournaud@u-psud.fr

\section{PATRICK PAMPHILE}

Laboratoire de Mathématiques d'Orsay (LMO), Université Paris Sud, Université Paris Saclay, patrick.pamphile@u-psud.fr 